\title{
Multi-criteria optimization of multi product assembly line using hybrid Tabu-SA algorithm
}

\author{
Ahmad Tavakoli ${ }^{1}$ (1) \\ Received: 5 August 2019 / Accepted: 7 December 2019 / Published online: 3 January 2020 \\ (c) Springer Nature Switzerland AG 2020
}

\begin{abstract}
In this paper, a multi-criteria optimization problem has been addressed including minimizing three important objectives: (a) total amount of fines for untimely production of orders; (b) total carrier rails' time including the preparation times of the stations; and (c) total cost of feeding outside the work station plan. In order to consider the relative importance of the stated goals, all costs/fines have been weighed. The proposed problem is one the nondeterministic polynomial complex problems, for which no comprehensive solution has been presented. Hence, to find the optimal answer in a reasonable time, Tabu search (TS) and simulated annealing (SA) algorithms have been used. Furthermore, to obtain the merits of the stated algorithms, a combination of both has been employed (Tabu-SA algorithm). To illustrate the performance of the proposed method, two numerical examples have been solved. The computational results indicate the high speed of integrated search algorithm and the superiority of the quality of its solutions. Based on the results, the proposed hybrid Tabu-SA algorithm has a remarkable advantage in comparison with TS and SA algorithms in terms of convergence rate. The total cost has been reduced at $24 \%$ using the proposed hybrid Tabu-SA algorithm. Besides, the computing time was improved at $29 \%$ and $46 \%$ using the proposed procedure respectively.
\end{abstract}

Keywords Multi-criteria optimization · Multi modal assembly lines (MMAL) · Tabu search (TS) algorithm · Simulated annealing (SA) algorithm

\section{Introduction}

Multi model assembly lines (MMALs) are lines in which a variety of products with similar production characteristics are assembled with a low volume. Of the most prominent examples of such production systems, multi-line assembly lines of the automotive industry can be mentioned, in which different car models with common infrastructures and various components (engine type, gearbox, etc.) are assembled.

Today, diverse customer demands and the possibility to offer a variety of choices in products such as automobiles, it has led to production and assembly strategies based on ordering and to implement lean inking. In this regard, the production and assembly lines of these products are designed and manufactured for a low volume production from each model with a wide variety.

Multi modal or flexible production lines usually have a higher technology in comparison with mass production lines, in which a relatively high investment is required. Therefore, in order to reduce costs, the production planning of such lines is of particular importance. In general, the purpose of production planning is to determine the type, number, sequence, and production times of the products, taking into account the resources required and the production commitments, which is usually done to reduce orders and costs in order to reduce production costs. For MMLAs, researchers have taken various

Ahmad Tavakoli, tavakoli-a@um.ac.ir|'Department of Management, Faculty of Economics and Administrative Sciences, Ferdowsi University of Mashhad, Mashhad, Iran. 
measures: minimizing cost of untimely production [1, 3], minimizing station preparation costs $[2,3]$, minimizing the total stopping time of line and idle time of workers [4], and minimizing the cost of feeding outside the work station [5, 6]. Each of these goals is of paramount importance; however, there has not yet been a comprehensive model that includes a combination of these goals simultaneously [7].

The complex nature of multi-criteria production models and the magnitude of real issues in the industry have led to standard methods in which solving speed and efficiency are not acceptable [1]. Nowadays, with the advancements in computer sciences, innovative methods such as heuristic algorithms including genetic, simulated annealing and Tabu search algorithms have been widely used in solving such complex problems. The principles of these algorithms are to create and evaluate a limited number of acceptable solutions in order to reach the optimal answer at acceptable times.

This research focuses on three important objectives: (1) Minimizing the penalties for untimely production; (2) minimizing the total carrier rails' time including the preparation times of the stations and (3) minimizing the total cost of feeding outside the work station plan. To solve this problem, Tabu search (TS) and simulated annealing (SA) algorithms and a combination of TS and SA algorithms (Tabu-SA algorithm) have been used. Moreover, numerical examples have been used to evaluate the adequacy of the proposed method. First, the structure and characteristics of the production system along with the objectives has been described and then, the algorithms and their function have been introduced. Finally, two numerical examples have been solved and the computational results have been presented.

\section{Literature survey}

An approach has been introduced by Lai et al. [8], using which the number of workstations used in an assembly line has been minimize. At the initial step of the procedure, a sufficient condition for an optimal line balance has been derived to be stable. Then, it has been illustrated that the stability radius of an optimal line balance could be infinitely large. Next, some lower and upper bounds for a finite stability radius have been established. Finally, formulae that were needed and developed an algorithm for obtaining the stability radius of an optimal line balance have been derived.

The car sequencing problem with consideration of changeover complexity for MMALs has been studied by Sun and Fan [9]. It was defined as the uncertainty of feature options assembled at different stations in a MMAL, with both static and dynamic aspects perceivable by operators.
For minimizing purposes of production rates, various information entropy based formulations and models have been developed to measure the levels of three categories of changeover complexities. The changeover complexity problem in which minimizing the number of capacity violations and minimizing the total changeover complexity were considered as the primary and secondary objectives respectively, was proposed. The dynamic aspect of changeover complexity is dependent on daily production sequences. To deal with and tackle the multi-criteria optimization problem, a lexicographic MOACO algorithm has been proposed. The performance of the proposed algorithm has been validated carrying out computational experiments comprising either simple or complex examples for which the algorithm has been applied. It has been demonstrated that the proposed MOACO algorithm can effectively address the requirements of the two objectives simultaneously. The complexity measures were developed and the work changeovers contents at various stations were incorporated, which are determined by production sequences and account for the dynamic aspect of complexity. The secondary objective of the proposed CSCC problem for reducing of the negative impact of manufacturing complexity purpose during car sequencing process is reduction of total changeover complexity. The efficiency of the proposed MOACO algorithm has presented the feasibility of mitigating changeover complexity on condition that work overload is minimized.

Finite capacity material requirement planning system known as FCMRP, is one of the most important NP-hard problems in industrial scale flexible flow shops. In this regard, a new hybrid algorithm for FCMRP system in a flexible flow shop with assembly operations has been proposed. Furthermore, a hybrid algorithm consist of genetic and Tabu search (HGATS) algorithms has been also introduced. The performance of the proposed algorithm (HGATS) has been controlled using GA and TS parameters. The proposed GA was coupled to a parametric simulator which contained the simulated throughput for evaluation of the objective function. Two alternative chromosomal representations were tested on an ample set of examples (from the literature). The best solutions have also been compared with the best solutions known in the literature, on the same instances, for straight lines with buffers and parallel workstations. Due to acting the crossover work centers similar to unitary buffers, providing two places in which two loads can be placed simultaneously, it could be concluded that U-shaped lines are generally more efficient in comparison with the straight lines with buffers. The superiority of U-shaped lines holds true as long as it is possible to take full advantage of the employment of crossover work centers. For particular types of instances, depending on the distribution of task times, this possibility decreases, in which straight lines with parallel work stations and buffers are preferable [10]. 
To deal with the mixed model assembly line balancing problem of type I (MMALBP-I), bees algorithm (BA) with some particular features of the real world problems such as parallel work stations, zoning constraints and sequence dependent setup times between tasks has been proposed. The group behavior of honey bees in a single and multiple colonies simulates has been introduced and developed in BA. As the multiple colony structure of the real honey bees, each colony represents the honey bees living in a different hive and is generated with a different heuristic algorithm. Moreover, the multiple colony type of BA is more realistic than the single colony type. In the proposed approach, the performance of the proposed multiple colony algorithm was tested on 36 representatives MMALBP-I extended by adding low, medium and high variability of setup times. The superior performance of the proposed multiple colony algorithm has been verified based on the computational results. The optimal solutions of some problems provided by MILP model have also been incorporated [11].

A mixed integer linear programming model has been introduced and developed to tackle balancing and sequencing problems in a MMAL of a continuously moving conveyor simultaneously. The main objectives of the proposed model were minimizing the length and number of workstations, the workstations and the tasks duplication costs. The GA has also been incorporated to overcome the computational difficulties in solving large size problems. In GA, a solution representation encodes the model sequence as well as task-assignment to different workstations. To illustrate the computational efficiency of the developed GA, numerical examples have been presented. Furthermore, the performance of the proposed GA has been checked using branch and bound (BB) algorithm. Based on the obtained results, the proposed GA outperformed $\mathrm{BB}$ algorithm in finding an acceptable solution in much less computational time which was close to the optimal solution [12].

\section{Problem definition}

As it has been already mentioned, the system is a multimodal assembly line. The requirements of any model such as tools, instructions, assembly times, parts and sub-sets, could be similar or different with various delivery times for orders. The workstations and their personnel are flexible and each station has the ability to perform various operations on different products. However, some of the items are only deployed at certain stations independent of other stations. Each workstation has a limited capacity for parts and assemblies; therefore, during each production shift, these stations may be fed frequently. Feeding stations in fixed time sequences or when needed, which is feeding outside the work station, include additional costs. Relatively, the structure and characteristics of the production planning problem can be described as follows:

1. The assembly line has $K$ stations of the same length, through which a fixed speed $(V c)$ carrier rail passing.

2. Each station has a limited capacity to hold parts and pre-assemblies of each model.

3. Stations are fed from parts in fixed time sequences (loading periods). The cost of this periodic feeding is constant (such as the cost of rail travel or the cost of workers' wages or etc.). However, during the production period, it is required to feed earlier than the time required, a charge for loading outside the program should be considered.

4. One or more workers are assigned to the workstation $(k=1,2 \ldots K)$, working only at the same station.

5. The time required for workers in a station to carry out the process on each product equals the total assembly time of the relevant parts of the station and the time to prepare the station for the next product on the rail.

6. If the workers of a station cannot complete their tasks (assembly of necessary parts and preparation of the station) to the lower boundary of the station, the carrier rail will stop. Clearly, the time required to stop the carrier rails is the same as the longest time required for stations. Stopping the line is undesirable and generates cost.

7. According to the received orders, the volume of production that should be produced in a given time period is known, and therefore, during each shift, the products from different models are placed on the rails and arrive at the assembly line from the first station.

Considering the above characteristics, the optimal production plan here is to determine the number, type and sequence of the products in responding orders with low possible cost. Also, the number of stop times and the corresponding times, the feeding times outside the work station plan, and the time required to prepare the products for each order are determined by the mathematical model of the problem. The production plan is designed to minimize the total weight of line stop costs, out-off-program feeding costs, and the costs of untimely production (both tardy and early).

\section{Designing a mathematical model}

In this section, an integer programming model, which contains problem characteristics and satisfies its objectives and constraints is being presented. The value of the objective function in this model is calculated from the form of its 
production plan. Therefore, the cost of running an application varies for each sequence.

\section{Variables}

In the definition of the following symbols, "upper indices" are a two-letter expression representing the parameter name, while each letter in the "lower indices" represents an independent variable.
Number of products in $k$ th order

Line stopping cost per time unit

Off- planning loading cost of the station $j$ from model $m$ (unusual loading)

$U_{i m r}= \begin{cases}1 & \text { If member } i \text { and }(i+1) \text { of the string belong to model } m \text { and } r \text { respectively } \\ 0 & \text { Otherwise }\end{cases}$

\section{Decision making variables}

\section{.}




$$
\begin{aligned}
\text { Min.f }= & \sum_{k=1}^{K} a b s\left\{\left(W_{k} \cdot p_{k}^{t a}+\left(1-W_{k}\right) \cdot p_{k}^{e a}\right) \cdot\left(t_{k}^{d d}-\sum_{i=1}^{1} R_{i k} \cdot t_{i}^{e x}\right)\right\} \\
& +\stackrel{s t}{p} \cdot \sum_{j=1}^{J} \sum_{i=1}^{1} \overline{t_{i j}^{s t}} \\
& +\sum_{j=1}^{J} \sum_{i=1}^{\prime} \sum_{m=1}^{M} X_{i m} \cdot C_{j m}^{u l}
\end{aligned}
$$

\section{S.T.}

$\overline{t_{i j}^{s t}}=\max \left(t_{(i+j-x) x}^{s t}\right) \quad \forall i \in l, \quad \forall j \in J, x=1, \ldots,(i+j-1)$

$t_{i j}^{s t}=\left(\sum_{m=1}^{M} V_{i m} t_{j m}^{p r}+\sum_{m=1}^{M} \sum_{r=1}^{M} U_{i m r} t_{j m r}^{s e}\right)-T \quad i \in l, j \in J$

$t_{i}^{e x}=t_{i j}^{e s}($ or $) t_{i}^{e x}=t_{i j}^{f s}+T+\overline{t_{i j}^{s t}}$

$t_{i j}^{f s}=\sum_{r=1}^{i-1}\left(T+\overline{t_{r 1}^{s t}}\right)+\sum_{s=1}^{j-1}\left(T+\overline{t_{i s}^{s t}}\right) \quad i \in I_{,} j \in J$

$t_{i j}^{e s}=\sum_{r=1}^{i-1}\left(T+\overline{t_{r 1}^{s t}}\right)+\sum_{s=1}^{j}\left(T+\overline{t_{i s}^{s t}}\right) \quad i \in l, j \in J$

$\sum_{m=1}^{M} \sum_{r=1}^{M} U_{i m r}=1 \quad \forall i$

$\sum_{m=1}^{M} V_{i m}=1 \quad \forall i$

$\sum_{i=1}^{I} \sum_{r=1}^{M} U_{i m r}=d_{m} \quad \forall m$

$\sum_{i=1}^{l} v_{i m}=d_{m} \quad \forall m$

$\sum_{i=1}^{l} R_{i k}=1 \quad \forall k$

$X_{i m}=V_{i m} S_{i m} Y_{i j} Z_{m j}$

$U_{i m r}, V_{i m}, S_{i m}, Y_{i j}, Z_{m j}, R_{i k}, X_{i m}=0$ or $1 \quad \forall i, j, k, m, r$

The cost function of the problem, Eq. (1), has been developed from sum of the four sentences: the first sentence, which is converted by the coefficient $p_{k}^{\text {ta }}$ into cost, represents the total penalty of tardiness; the second sentence, which is converted by coefficient $p_{k}^{\text {ea }}$ into cost, represents the total penalty of earliness; the third sentence, which is converted by the coefficient $p$ into cost, indicates the cost of stopping the line and finally the last sentence indicating the cost of off-plan loading.

Equation (2) indicates the fact that the time at which the carrier rails are stopped is equal to the maximum time required by the workers at the station, where the model is being located. Equation (3), is the mathematical model for hypotheses 5 and 6 . Equations (4), (5), and (6), calculate the waiting times for the complete assembly of the specified part of string, the arrival of that member (part) at the beginning of the specified station and the arrival of that member at the end of that station respectively. The expression $\sum_{r=1}^{i-1}\left(T+\overline{t_{r 1}^{s t}}\right)$ in the above equations represents the time of waiting for the member $i$ to reach the beginning of the first station; the expression $\sum_{s=1}^{j-1}\left(T+\overline{t_{i s}^{s t}}\right)$ suggests the required time for the member $i$ of the string to reach the beginning of the first station to the beginning of station $j$ or to the end of station $(j-1)$. Equations (7) and (8) show that each member of the string contains only one model (or product). Equations (9) and (10) guarantee that the demand is satisfied from each model. Equation (11) has two meanings: a) ensures that all orders are completed; and b) only one member of the discipline completes $k$ th order. In Eq. (12), when the value of the correct variable $X_{i m}$ equals 1 , loading is done outside of the program and this is when all the variables on the second side equal 1.

\section{Designing the solution method}

There is a plenty of different heuristic algorithms (including Tabu search, GA, SA, PSO, Ant and Bee colony, and etc.) among which SA and Tabu search algorithms based on their merits are employed extensively for optimization of different problems and processes [13-18]. Easy to program and converge fast are the significant advantages of using TS algorithm. GA is another widely used algorithm coding which is a time consuming process due to setting its large number of parameters. SA's major merit over other algorithms is its ability to avoid getting trapped in local minima. As with GA a major advantage of SA is its flexibility and robustness as a global search method. SA algorithm does not use gradient information and makes relatively few assumptions about the problem being solved. It can deal with highly nonlinear problems and no differentiable functions as well as functions with multiple local optima. 
SA is a very powerful and important tool in a variety of disciplines.

Based on the above mentioned reasons, in this research, SA and TS algorithms have been introduced and integrated Tabu-SA algorithm has been derived from the combination of TS and SA algorithms. Also, after describing TS and $S A$ algorithms, the proposed hybrid algorithm will be presented.

\section{Tabu search algorithm}

Tabu search (TS) method which known as TS algorithm has been first proposed by Glover and Laguna [19]. TS algorithm is a local search method in which the chance of reaching a global extreme has been modified, and the possibility of increasing the value of the objective function in consecutive algorithm iterations has also been increased. In different algorithms it may happen some parts of solution space are searched by algorithm in a series of multiple times. In order to avoid this phenomenon, and enable the algorithm to jump over the local optimum, a Tabu mechanism has been introduced. A neighborhood is generated (in which the forbidden solutions by Tabu mechanism are not considered), unless the objective criteria has been met (i.e. the conditions under which the Tabu constraints may be omitted) $[19,20]$. The two-to-two displacement is the most commonly used neighboring mechanism in TS algorithm in which each new neighbor (new answer) moves from two processes (or products) to the current answer. Thus, the number of neighboring results of a sequence with $M$ members will be equal to $M(M-1) / 2$, and for each movement this number of answers must be generated and evaluated.

\section{Simulated annealing algorithm}

There are some heuristic algorithms whose operation is reminiscent of natural and physical phenomena, among which, simulated annealing (SA) algorithm first proposed By Kirkpatrick (1983), is being excessively used for optimization purposes in different industrial aspects [19]. SA, is a local-based search algorithm is being employed when global optimum is hidden among many local goals for tackling complex problems. The method itself has a direct similarity with annealing process used for thermal treatments, in which a solid is first heated up to a high temperature and then cooled down slowly until it reaches a thermodynamic equilibrium. Therefore, the absolute minimum level of energy is being achieved, when the temperature at a slow rate is being condensed. The process in which the temperature increased up to a specific value and then reduces at a slow rate is known as annealing process. As the same token, in TIG welding process, a minimum energy function is created. While it has been tried to avoid local and achieve global minima. Nowadays, SA algorithm has been emerged as a leading tool for large-scale combinational optimization problems.

Firstly, in standard SA procedure, a preliminary point has been set and the value of the objective function is being computed. Then, a small random change is made in the current solution (answer). Next, the objective function value of the new solution is calculated and compared with that of the current one. A move to the new solution will be made, only if it has better value or the probability function implemented in SA (Eq. 14) has a higher value than a randomly generated number between $(0,1][20]$ :

$P_{r}\left(\right.$ Solution_current ${ }_{k+1}=$ Solution_neighbour $\left.{ }_{k}\right)=\exp \left(-\frac{\Delta C}{C_{k}}\right)$

In Eq. (14), the difference between the current and new answers of the target function has been shown by $\Delta C$. The index $k$ is the number of repetitions (iteration) and the control parameter $c_{k}$ called temperature. Based on the Eq. 14, at the beginning of the searching process, the initial temperature value $c_{0}$ is chosen, so that the algorithm has a greater chance of movement due to the higher value of it. Therefore, at the initially stages the worsening answers would have been accepted. But with increasing the number of moves (iterations), this temperature gradually decreases (according to a cooling schedule function); hence, the probability of accepting worse solutions (answers) with increasing number of moves decreases. In other words, at the beginning of the search, the role of the random nature of the algorithm in accepting new neighborhoods is more important than the role of its definite nature. But as search proceeds, the moves are more based on the improvement of the target function and the role of the random nature of the algorithm in accepting the new answer decreases. This approach would help the algorithm escaping from the local optimum by jumping over it. The extensive explanation of the above algorithms has been well documented in the references $[20,21]$.

\section{Tabu-SA integrated algorithm}

Based on TS and SA algorithms principles, each algorithm has its own advantages and disadvantages. The unique feature of TS is escaping the local optimizations and the legitimate nature of this search engine can be considered as one of the most important benefits. In problems 
of optimization, especially in small ones and the final stages of searching for big ones, these two features play a major role in improving the quality of the final answer. Nevertheless, in big problems, maintaining the definite nature of an algorithm that results from the evaluation of all neighborhoods of the current answer, will slow down the progression and extend the computing time. On the other hand, the SA algorithm has a high speed due to its random nature in assessing and selecting neighborhoods and is also able to cover a wider range of acceptable solutions. However, the risk of returning and repeating previous routes, as well as its inability to escape local optimizations at low temperatures, are subject to limitations [22].

The proposed Tabu-SA algorithm is a combination of TS and SA algorithms that have been used to design SA's speed in the generation and evaluation of new neighborhoods, and the prohibited TS rules and regulations have been used to guide the algorithm to the optimal solution at the same time. For this purpose, the generation and assessment of neighborhood in Tabu-SA algorithm is based on SA algorithm. However, this algorithm is also equipped with a Tabu list in order not to return to previous paths. Another point that has been considered in the design of Tabu-SA algorithm is to create a mechanism for preventing the algorithm to be trapped in local optimizations at the late stages of the search. As the temperatures decreases, SA algorithm only moves to new answers that are better than the current ones, and the probability of choosing worse answers decreases gradually. If the current answer is a local optimum, the algorithm may not be able to find a better answer at the neighborhood. Hence, the algorithm will be trapped in local optimum point. If the algorithm after the generation and evaluation of a certain number of neighborhoods is unable to select any of them, the mechanism regarding the formation and selection of the answers to TS will change, in order to allow the algorithm to escape the current trap. A summary of the proposed algorithm has been given step by step as follows:
Step 0 Receive problem inputs, search parameters, and create an acceptable production plan as the starting point.

Step 1 Generate a new neighborhood randomly;

Step 2 Assess and compute the cost of the neighboring solution;

Step 3 Check the acceptance conditions for the new answer; if the cost of the new production program is less than the current answer or the probability of accepting a worse response, more than one random number in the interval $([0,1)$, the algorithm will refer to step 5), otherwise it returns to step 1 ; and if the number of these returns increases to a greater extent, the algorithm is caught up in the optimal local trap and it goes to the next step.

Step 4 Get rid of local optimal traps. In this step, the algorithm uses TS algorithm and moves to the best answer in the neighborhood of the current answer.

Step 5 Check whether there is a new answer on Tabu list; if the new answer is in the Tabu list, the algorithm returns to step 1; otherwise, it will go back to the next step.

Step 6 Move the algorithm to the new answer and update the search parameters and the answers in Tabu list.

Step 7 Check the stop criteria. If the stop criteria are not met, the algorithm returns to step 1 , otherwise it will announce the search stop and provide the required outputs.

In general, before the start of the search, the inputs of the problem and the parameters of the algorithm should be given to the algorithm and to the start point. The next steps include building a new neighborhood, checking the conditions, and updating the search parameters.

Table 1 Feeding outside the work station

\begin{tabular}{|c|c|c|c|c|c|c|c|c|c|c|c|c|}
\hline Models & A & & B & & $\mathrm{C}$ & & & $\mathrm{D}$ & & $\mathrm{E}$ & & \\
\hline Number of stations needed for each model & 1 & 3 & 1 & 2 & 1 & 2 & 3 & 2 & 3 & 1 & 2 & 3 \\
\hline Maximum station capacity (number) & 1 & 1 & 2 & 1 & 2 & 1 & 3 & 2 & 1 & 1 & 2 & 2 \\
\hline Expenditure on nutrition outside the program (dollars) & 10 & 15 & 25 & 30 & 25 & 30 & 10 & 20 & 20 & 20 & 25 & 20 \\
\hline
\end{tabular}

Table 2 Production obligations

\begin{tabular}{lrrrrrrrrr}
\hline Orders & A1 & A2 & B1 & B2 & C1 & C2 & D1 & D2 & E1 \\
\hline Delivery time (min) & 40 & 80 & 50 & 90 & 40 & 80 & 60 & 110 & 100 \\
Number & 2 & 3 & 1 & 4 & 1 & 2 & 3 & 2 & 2 \\
\hline
\end{tabular}


Table 3 The results obtained by solving example 1 with two different solution methods

\begin{tabular}{|c|c|c|c|c|c|}
\hline \multirow[t]{2}{*}{ Methods } & Costs & & & & \\
\hline & Total cost (\$) & Untimely fines (\$) & Rail stop fines (\$) & $\begin{array}{l}\text { Cost of out-of-pro- } \\
\text { gram feeding (\$) }\end{array}$ & $\begin{array}{l}\text { Comput- } \\
\text { ing time }\end{array}$ \\
\hline
\end{tabular}

(s)

\begin{tabular}{lllllr}
\hline Tabu-SA & 871.95 & 656.47 & 35.48 & 180 & 65 \\
Counting & 857.31 & 654.31 & 23 & 180 & 17,629 \\
\hline
\end{tabular}

Table 4 Specifications and costs of assembly line in example 2

\begin{tabular}{|c|c|c|c|c|c|c|c|c|}
\hline \multirow{2}{*}{$\begin{array}{l}\text { Number of stations } \\
\text { required for each } \\
\text { model (number) } \\
40\end{array}$} & \multicolumn{2}{|c|}{$\begin{array}{l}\text { Length of each sta- } \\
\text { tion }(\mathrm{m})\end{array}$} & \multicolumn{2}{|c|}{ Carrier speed (m/min) } & \multicolumn{2}{|c|}{$\begin{array}{l}\text { Number of models } \\
\text { (number) }\end{array}$} & $\begin{array}{l}\text { Number of orders } \\
\text { (number) }\end{array}$ & \multirow{2}{*}{$\begin{array}{l}\text { Number of stations } \\
\text { (number) }\end{array}$} \\
\hline & 10 & & 1 & & 5 & 15 & & \\
\hline $\begin{array}{l}\text { Running cost stop } \\
\text { coefficient (dollars } \\
\text { per minute) }\end{array}$ & $\begin{array}{l}\text { Loading period } \\
\text { (min) }\end{array}$ & $\begin{array}{l}\text { Num } \\
\text { ucts } \\
\text { (num }\end{array}$ & $\begin{array}{l}\text { er of prod- } \\
\text { quested } \\
\text { er) }\end{array}$ & $\begin{array}{l}\text { Preparatior } \\
\text { (min) }\end{array}$ & n times & $\begin{array}{l}\text { Production times } \\
\text { (minutes) }\end{array}$ & $\begin{array}{l}\text { Capacity of sta- } \\
\text { tions (number) }\end{array}$ & $\begin{array}{l}\text { Cost of feeding } \\
\text { off the schedule } \\
\text { (dollars) }\end{array}$ \\
\hline 1000 & 25 & 1000 & & $0.5-3$ & & $5-9$ & $1-4$ & $10-20$ \\
\hline
\end{tabular}

\section{Examples and computational results}

In this section, to discuss the performance of the proposed algorithm in solving production planning problems, a trivial example is presented, and then a computational summary is presented for a real sized problem. Example 1 , here, a multi model assembly line with 3 workstations is considered for production of 5 different models A, B, $C, D$ and $E$. Each model requires at least two specific stations, and the capacity of the stations to store parts is limited. This information, along with the cost of feeding outside the station program, is given in Table 1. Moreover, Table 2 contains information on production commitments (orders), which are timely deductible penalties of between 0.5 and 2.5 (dollars per minute). In this table, for example, product $\mathrm{A}$ has two orders of $\mathrm{A} 1$ and $\mathrm{A} 2$ for 2 and 3 , respectively, for delivery times of 40 and $80 \mathrm{~min}$ (or each other time). As a result, the total demand (produced goods) will be 20 . The preparation times of the stations are from 2 to $4.5 \mathrm{~min}$ and the time of each process in each station is between 5 and $10 \mathrm{~min}$. Planned feeds are done every $20 \mathrm{~min}$. The objective function, which includes delays, line stops and out-of-station feeding, is expressed in terms of currency (for example, dollar). The penalty is 10 dollars per minute.

The number of possible sequences (production programs) for the above problem is equal to 9 ! $(362,880)$, in order to verify the accuracy of the algorithm, all possible states were solved and the optimal sequence was determined. The time taken for this survey was about $5 \mathrm{~h}$ and the cost of the optimum production program was 857.31

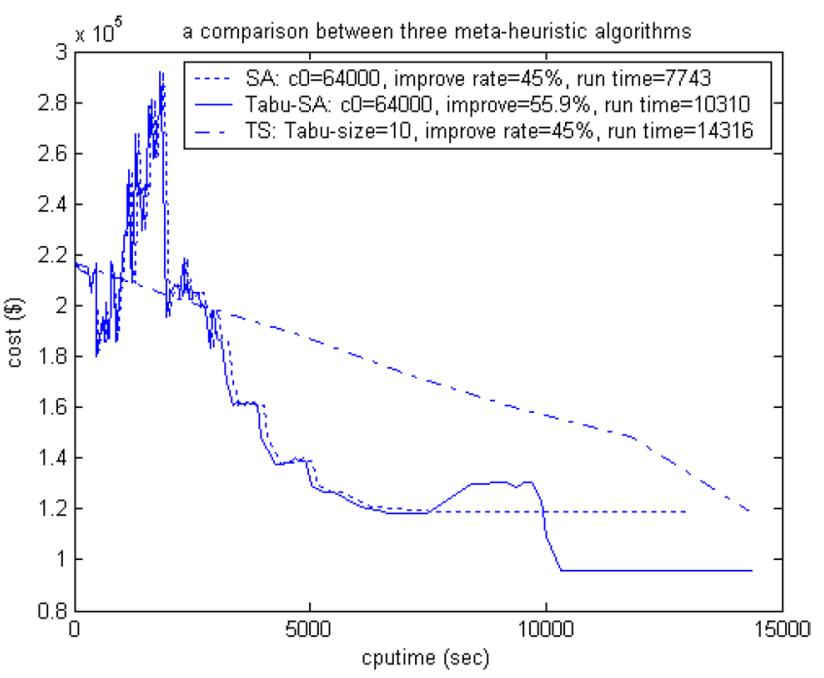

Fig. 1 Convergence of the proposed algorithms for solving example 2

dollars, as the problem grows, the time required to count the sequences will increase exponentially. The optimal solution of the above problem with the proposed TabuSA algorithm lasted slightly longer than $1 \mathrm{~min}$. The optimal production plan and the details of the costs are given in Table 3.

Example 2 Solving a great example: In this section, the production of a car assembly line with 40 workstations and 5 models has been investigated. Regarding production planning, 1000 different cars must be produced from different models for 15 different time periods ( 15 different 
Table 5 Results of solving example 2 using TS, SA and Tabu-SA algorithms

\begin{tabular}{|c|c|c|c|}
\hline \multirow[t]{2}{*}{ Results } & \multicolumn{3}{|l|}{ Algorithms } \\
\hline & $\begin{array}{l}\text { Tabu search } \\
\text { (TS) }\end{array}$ & $\begin{array}{l}\text { Simulated } \\
\text { Annealing } \\
\text { (SA) }\end{array}$ & Hybrid (Tabu-SA) \\
\hline Total costs $(\$)$ & $119,180.3$ & $119,182.5$ & $95,529.1$ \\
\hline $\begin{array}{l}\text { Total costs } \\
\text { reduction }\end{array}$ & 45 & 45 & 55.9 \\
\hline $\begin{array}{r}\text { Computing } \\
\text { time (sec) }\end{array}$ & 14,316 & 7743 & 10,310 \\
\hline $\begin{array}{l}\text { Tardiness fines } \\
\text { (\$) }\end{array}$ & $28,336.8$ & $32,384.9$ & $17,386.7$ \\
\hline $\begin{array}{l}\text { Earliness fines } \\
(\$)\end{array}$ & $10,300.9$ & $12,633.6$ & 7455.5 \\
\hline $\begin{array}{l}\text { Cost of rail stop } \\
(\$)\end{array}$ & $64,672.3$ & $58,269.4$ & $54,844.6$ \\
\hline $\begin{array}{l}\text { Out of produc- } \\
\text { tion plan } \\
\text { feeding cost } \\
(\$)\end{array}$ & $15,870.3$ & $15,894.6$ & $15,842.3$ \\
\hline
\end{tabular}

orders) should be produced. The above information is similar to the actual specifications of the world's automotive factories. All specification and costs of the problem are given in Table 4.

This problem is solved with TS, SA and Tabu-SA algorithms, and the convergence of the algorithms has been shown in Fig. 1. As it can be seen from this diagram, the proposed Tabu-SA algorithm has a remarkable advantage in comparison with TS algorithm in terms of convergence rate. Also, the comparison of SA and Tabu-SA shows that, under similar conditions, the convergence rate of the proposed algorithm is slightly higher, and the proposed model can avoid local optimizations. Based on the information given in Table 5, all the items have been developed using hybrid algorithm in comparison with SA and TS algorithms. In this regard, the total cost has been reduced at $24 \%$ using the proposed hybrid algorithm. Furthermore, time of computing has been improved using SA and Tabu-SA at $29 \%$ and $46 \%$ respectively. Details of the results are presented in Table 5 .

\section{Conclusion and discussion}

Today, the diversity of products such as cars and required rapid responses from these industries to the market and customers' requests, have all made the production planning of these products very important. The absence of an optimized production planning leads to an increased production cost. In this research, a relatively comprehensive model of multi modal assembly lines has been presented.
Timely production, limited capacity of workstations and line stop costs are among the important factors that are considered for the first time in this research simultaneously. This problem was solved using Tabu search (TS), simulated annealing (SA) and hybrid Tabu-SA algorithms. According to the results of these three algorithms, the proposed Tabu-SA method has a significant advantage over the convergence rate compared to TS and SA algorithms. Furthermore, Tabu-SA algorithm can escape local optimums. Based on the results, the hybrid Tabu-SA algorithm has the ability to solve complicated multi-criteria optimization problems efficiently. Therefore, due to the adaptability and flexible structure of this algorithm, it could be expected that this algorithm solves other optimization models at reasonable times. Furthermore, the proposed modeling and optimization approach, with minor changes, can be carried out using different algorithms and procedures such as Emperor penguin optimizer, seagull optimization algorithm and spotted hyena optimizer. Furthermore, using well-known benchmark test functions can act as a tool to validate the performance of the proposed procedure.

\section{Compliance with ethical standards}

Conflict of interest The authors declare that they have no conflict of interest.

\section{References}

1. McMullen PR (2001) An efficient frontier approach to addressing JIT sequencing problems with setups via search heuristics. Comput Ind Eng 41(12):335-353

2. Azizoğlu M, İmatb S (2018) Workload smoothing in simple assembly line balancing. Comput Oper Res 89(21):51-57

3. McMullen PR (2001) An ant colony optimization approach to addressing a JIT sequencing problem with multiple objectives. Artif Intell Eng 15(2):309-317

4. Xiaobo Z, Ohno K (2000) Properties of a sequencing problem for a mixed model assembly line with conveyor stoppages. Eur J Oper Res 124(5):560-570

5. Brudett RL, Kozan E (2000) Evolutionary algorithms for flow shop sequencing with non-unique jobs. Int Trans Oper Res 7:401-418

6. Hadi M, Zandieh M (2013) An imperialist competitive algorithm for a mixed-model assembly line sequencing problem. Journal of Manufacturing Systems 32:46-54

7. Neumann D, Keidel J (2019) A problem design and constraint modelling approach for collaborative assembly line planning. Robotics and Computer-Integrated Manufacturing 55(5):199-207

8. Lai TC, Sotskov YN, Dolgui A (2019) The stability radius of an optimal line balance with maximum efficiency for a simple assembly line. Eur J Oper Res 274(2):466-481 
9. Sun $\mathrm{H}$, Fan $\mathrm{S}$ (2018) Car sequencing for mixed-model assembly lines with consideration of changeover complexity. J Manuf Syst 46(4):93-102

10. Tiacci L (2017) Mixed-model U-shaped assembly lines: balancing and comparing with straight lines with buffers and parallel workstations. J Manuf Syst 32(1):102-112

11. Akpinar S, Baykasoglu A (2014) Modeling and solving mixedmodel assembly line balancing problem with setups. Part II: a multiple colony hybrid bee's algorithm. J Manuf Syst 33(4):445-461

12. Defersha FM, Mohebalizadehgashti F (2018) Simultaneous balancing, sequencing, and workstation planning for a mixed model manual assembly line using hybrid genetic algorithm. Comput Ind Eng 119(6):370-387

13. Azadi Moghaddam M, Golmezerji R, Kolahan F (2017) Simultaneous optimization of joint edge geometry and process parameters in gas metal arc welding using integrated ANN-PSO approach. Sci Iran B 24(1):260-273

14. Azadi Moghaddam M, Golmezerji R, Kolahan F (2016) Multi-variable measurements and optimization of GMAW parameters for API-X42 steel alloy using a hybrid BPNN-PSO approach. Measurement 92(5):279-287

15. Akram K, Kamal K, Zeb A (2016) Fast simulated annealing hybridized with quenching for solving job shop scheduling problem. Appl Soft Comput 49(6):510-523

16. Poursabzi O, Mohammadi M, Naderi B (2018) An improved model and a heuristic for capacitated lot sizing and scheduling in job shop problems. Sci Iran E 25(6):3667-3684
17. Pereira J, Rittb M, Vásquezc ÓC (2018) A memetic algorithm for the cost-oriented robotic assembly line balancing problem. Comput Oper Res 88(7):249-261

18. Sahu A, Pradhan SK (2018) Comparative analysis and optimization of mixed model assembly line using genetic algorithm. Materialstoday Proc 5(11):25075-25084

19. Kolahan F, Liang M (1998) An adaptive TS approach to JIT sequencing with variable processing times and sequencedependent setups. Eur J Oper Res 109(21):142-159

20. Henderson D, Jacobson SH, Johnson AW (2003) The theory and practice of simulated annealing. In: Handbook of metaheuristics, International series in operations research \& management science, vol 57(5). Springer, Boston, MA, pp 287-319

21. Zolfaghari A, Goharimanesh M, Akbari AA (2015) Optimum design of straight bevel gears pair using evolutionary algorithms. J Braz Soc Mech Sci Eng 39(6):2121-2129

22. Hamtaa N, Fatemi Ghomia SMT, Tavakkoli-Moghaddamb R, Jolaib F (2014) A hybrid meta-heuristic for balancing and scheduling assembly lines with sequence-independent setup times by considering deterioration tasks and learning effect. Sci Iran E 21(3):963-979

Publisher's Note Springer Nature remains neutral with regard to jurisdictional claims in published maps and institutional affiliations. 